\title{
Role of bone patellar tendon allograft in revision ACL reconstruction
}

\author{
Sukesh Rao Sankineani • Ramakanth Kumar • \\ Vijay Kumar · Ram Prasad Kancherla
}

Received: 21 February 2012/Published online: 24 April 2012

(C) Springer-Verlag 2012

We read with great interest the article by Hermann et al. [1] comparing the outcomes of allograft and allograft patellar tendon graft in revision ACL reconstruction. We would like to congratulate the authors in elucidating in detail the potential benefits of use of allografts. We also agree with the authors that the use of allografts could be associated with significant reduction in morbidity. Furthermore, reduced donor harvest time, decreased tissue handling, and customizing bone blocks are easier with allograft than autograft [3]. However, we would like to raise some queries.

1. The possibility of bias in this study has been not ruled out as the authors have not commented whether there was any significant difference in the preoperative characteristics between the groups (randomization was not done in this study).

2. The comparison of parameters in the postoperative period was done at a broad range of time (e.g., $18.2 \pm 4.2$ months after operation for the allograft group, and $20.2 \pm 7.1$ months after operation for the autograft group).We believe that comparison at such irregular intervals would not accurately assess the clinical outcome as there will be variability in the graft strength at different due to the process of graft incorporation into the host tissue environment [3].
3. The patients in the autograft group had a prior ACL reconstruction with hamstring tendons. These patients are likely to have alteration in the biomechanics of knee [2]. Though not yet investigated, we believe this could likely to influence the clinical outcome and error in interpretation.

4. We do agree with the authors that the sample size is small and that concurrent procedures on the meniscus would lead to an alteration in the rehabilitation protocol and influence the outcome [1, 3].

We hope that the authors would find our comments useful in the context of their study and we wish them all the best wishes.

\section{References}

1. Mayr HO et al. Revision of anterior cruciate ligament reconstruction with patellar tendon allograft and autograft: 2-and 5-year results. Arch Orthop Trauma Surg. doi:10.1007/s00402-012-1481-z (Online first 16 Feb. 2012)

2. Soon M, Neo CPC, Mitra AK, Tay BK (2004) Morbidity following anterior cruciate ligament reconstruction using hamstring autograft. Ann Acad Med Singap 33(2):214-219

3. Smith HA, Bach RB Jr, Bush-Joseph AC (2005) Allograft for revision ACL reconstruction: the RUSH experience. Sports Med Arthrosc Rev 13(2):86-92 